\title{
Theoretical model of the helium pinhole microscope
}

\author{
Adrià Salvador Palau, ${ }^{1}$ Gianangelo Bracco, ${ }^{1,2}$ and Bodil Holst ${ }^{1, *}$ \\ ${ }^{1}$ Department of Physics and Technology, University of Bergen, Allégaten 55, 5007 Bergen, Norway \\ ${ }^{2}$ CNR-IMEM, Department of Physics, University of Genova, V Dodecaneso 33, 16146 Genova, Italy
}

(Dated: November 18, 2016)

\begin{abstract}
In recent years, the development of neutral helium microscopes has gained increasing interest. The low energy, charge neutrality and inertness of the helium atoms makes helium microscopy an attractive candidate for the imaging of a range of samples. The simplest neutral helium microscope is the so called pinhole microscope. It consists of a supersonic expansion helium beam collimated by two consecutive apertures (skimmer and pinhole), which together determine the beam spot size and hence the resolution at a given working distance to the sample. Due to the high ionization potential of neutral helium atoms, it is difficult to build efficient helium detectors. Therefore, it is crucial to optimize the microscope design to maximize the intensity for a given resolution and working distance. So far this has not been addressed in the literature. Here we present an optimisation model for the helium pinhole microscope system. We show that for a given resolution and working distance there is a single intensity maximum. Further we show that with present day state of the art detector technology (ionisation efficiency $1 \cdot 10^{-3}$ ), a resolution of the order of $600 \mathrm{~nm}$ at a working distance of $3 \mathrm{~mm}$ is possible. In order to make this quantification we have assumed a Lambertian reflecting surface and calculated the beam spot size that gives a signal $100 \mathrm{cts} / \mathrm{s}$ within a solid angle of $0.02 \pi \mathrm{sr}$, following an existing design. Reducing the working distance to the micron range leads to an improved resolution of around $40 \mathrm{~nm}$.
\end{abstract}

\section{INTRODUCTION}

Neutral helium microscopy, short Nemi or SHem (scanning helium microscopy), refers to the use of a beam of neutral helium atoms as an imaging probe. The neutral helium beam is created by a supersonic expansion and has a very low energy (typically less than $0.1 \mathrm{eV}$ ), which means that the atoms map the outermost electron density distribution of the sample and do not penetrate into solid material $[1,2]$. This makes neutral helium microscopy particularly suited for the investigation of fragile and/or insulating materials, nano-coatings and surfaces with high aspect ratios. By using two detectors it should even be possible to create a stereo microscope on the nano scale. Alternatively a small working distance helium microscope can be used to investigate all samples presently examined with scanning probe techniques.

The first neutral helium microscope images were published in 2008 [3]. They were transmission, shadow images of a porous structure. The images were obtained by using a Fresnel zone plate to focus a beam down to $3 \mu \mathrm{m}$ and later to less than $1 \mu \mathrm{m}$, scan the beam across the sample and record the transmitted intensity [4]. Early focussing experiments using neutral helium atoms were carried out by O.Carnal et al, Holst and Allison and Doak et al. in the 1990's [5-7]. Since then two other research groups have managed to obtain helium microscope images in reflection with micron range resolution using pinhole microscopes [8-11]. The first reflection images were published in 2011 by Withman and Sanchez using a setup with just a pinhole and no skimmer [8]. This setup still

\footnotetext{
* Corresponding author, Bodil.Holst@uib.no
}

claims the best resolution achieved so far with a helium microscope: $350 \mathrm{~nm}$ [12]. Mathematically the WithamSanchez setup can be seen as a special case of the two aperture microscope model we present in this paper, with the skimmer being very big. We therefore do not discuss it further.

A diagram of a helium pinhole microscope can be found in Fig. 1. The basic idea is simple: the supersonic expansion beam is collimated by two consecutive apertures, which we refer to as skimmer and pinhole. The pinhole is placed after the skimmer and is kept at a certain working distance from the sample plane. The resolution of the microscope is then determined by the spot size of the beam on the sample plane. In this work we define the resolution as the full width at half maximum intensity of the beam spot. The beam scattered off the sample is then collected at a given solid angle using a detector, and the variation in this signal, while the sample is scanned, is used to create an image.

The aim of this paper is to determine the geometry of the pinhole system which gives the maximum signal intensity in the beam spot on the sample for a given resolution. Our basic assumption is that the beam is created in a supersonic expansion. In such an expansion, the helium gas expands into vacuum through a nozzle that must have a diameter much bigger than the mean free path of the gas particles. The atoms then collide with each other until eventually collisions cease and the atoms are travelling in free molecular flow without interacting. The central part of the beam is selected by a collimating aperture (skimmer), which is conically-shaped to reduce backscattering of atoms into the beam. The supersonic expansion is chosen over for example an effusive source because it gives the highest centre line intensity [13].

A common way to describe a supersonic expansion the- 
oretically is the quitting surface model. Here, the spherical quitting surface represents the distance from the nozzle where the atoms have reached molecular flow and are no longer interacting [14]. The velocity distribution of the atoms along the surface can be described by the most probable velocity $\bar{v}$ along the parallel direction (meaning the radial direction from the centre of propagation). This velocity is given together with either a single parallel temperature or, in a more detailed description, by a pair of temperatures $T_{\|}, T_{\perp}$ associated to the orthogonal components of the velocity in spherical coordinates. At the quitting surface, the perpendicular temperature, $T_{\perp}$ must be much smaller than the parallel temperature $T_{\|}$. In this paper, the conditions at the quitting surface are calculated solving Boltzmann equation [15-17]. Negligible collisional coupling is assumed at a distance where the temperatures of the beam fulfil $T_{\perp} / T_{\|} \leq 0.01$. Stopping the integration at $T_{\perp} / T_{\|}=0.005$ was shown to affect the flow parameters by less than $0.1 \%$ with respect to the proposed setting $T_{\perp} / T_{\|} \leq 0.01$ [18].

For a single temperature and constant density along the quitting surface, an analytical approximation for the intensity exists, obtained by Sikora in 1973 [14]. For a pair of temperatures, a numerical integral must be implemented [19]. From now on, we will name the single temperature solution Sikora's approximation and, following the convention in literature, the dual temperature model ellipsoidal quitting surface model. Both models are explained in detail in Section II.

In practise, one will often measure a reduction in the signal intensity compared to the theoretical model due to backscattering of atoms into the beam. For the most efficient microscope, an optimization of the expansion parameters (distance between nozzle and skimmer, beam pressure and temperature etc.) is therefore important. This has been studied for example in [20-22], and is not a topic of this paper. Hence, in this work all the parameters relative to the expansion enter as constants in the equations: most probable velocity and perpendicular and parallel temperatures of the beam, distance between the skimmer and the nozzle and the position of the quitting surface relative to the skimmer.

To simplify the calculations we consider not only the resolution but also the working distance as a constant of the optimization; The behaviour of the system with respect to the working distance is monotone and easy to calculate if need be. From an experimental point of view the working distance is an important parameter. The larger the working distance the more flexible the microscope will be with respect to what kind of samples can be investigated.

This leaves us with three variable parameters as can be seen from Fig. 1: The aperture openings, $r_{\mathrm{S}}$ and $r_{\mathrm{ph}}$, and the distance between them, $a$. Once the desired resolution and working distance have been chosen, the system is reduced to a two variable optimization problem using the optical equations of the system, which we describe in the next section. The intensity can then be calculated over a wide span of combinations and plotted in a single graph. A single clear maximum is found that gives the best theoretical design of a pinhole helium microscope. In praxis it may be necessary to configure the microscope away from the maximum due to technical constraints. The calculations clearly show how much signal is lost compared to the maximum and provide the best choice from the subset of realisable microscopes.

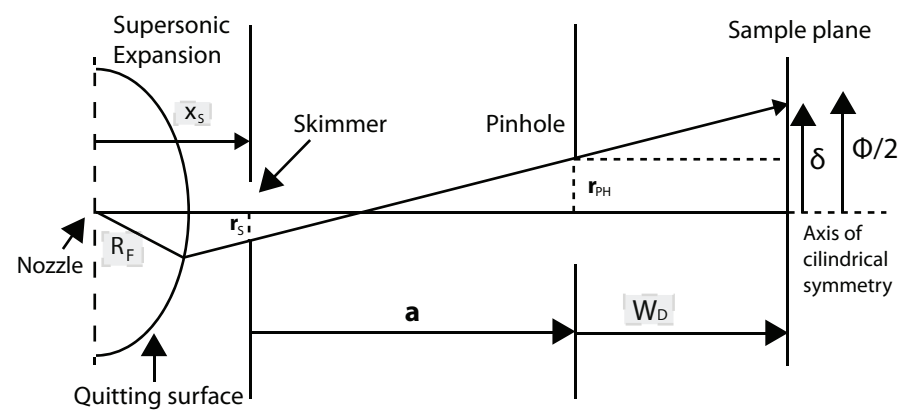

FIG. 1. Simplified illustration of a pinhole microscope setup. The constants of the problem are marked in grey boxes. $W_{\mathrm{D}}$ is the working distance, $\delta$ is the geometrical spread of the beam, $\Phi$ is the focal spot size. Due to diffraction effects $2 \delta$ is not always equal to $\Phi$. $r_{\mathrm{S}}$ and $r_{\mathrm{ph}}$ are the radius of the skimmer and the radius of the pinhole respectively and $a$ is the distance between the skimmer and the pinhole. Note that the system is cylindrically symmetric about the main axis.

\section{THEORETICAL FOUNDATION}

\section{A. The optical system}

The expression for the spread of the beam depends on the optical regime of the system, determined by the Fresnel number, F [23]:

$$
\mathrm{F}=\frac{r_{\mathrm{ph}}^{2}}{W_{D} \lambda} .
$$

Where $r_{\mathrm{ph}}$ is the radius of the pinhole and $W_{\mathrm{D}}$ is the working distance (see Fig. 1). $\lambda$ is the average de Broglie wavelength of the beam, given by $\lambda=h / m \bar{v} . m$ is the mass of a helium atom and $\bar{v}$ is the most probable velocity of helium atoms along the radial direction.

An analytical expression can be easily found that incorporates the two extreme optical cases: Geometrical optics $(\mathrm{F} » 1)$ and Fraunhofer diffraction $(\mathrm{F} \ll 1)$. We will see that for most real designs this expression suffices to determine the dynamics of the system. Using simple rayoptics, the geometrical image of the aperture projected onto the sample plane can be found to be:

$$
\delta=r_{\mathrm{ph}}\left(1+\frac{W_{\mathrm{D}}}{a}\right)+\frac{W_{\mathrm{D}} r_{\mathrm{S}}}{a},
$$

where $a$ is the distance between the skimmer and the pinhole and $r_{\mathrm{S}}$ is the radius of the skimmer. To obtain 
the standard deviation, $\sigma$, of the beam intensity profile we must convolute the geometrical image of the aperture with the Point Spread Function (PSF) of the diffraction trough the aperture. We know that the PSF of the Fraunhofer diffraction across a circular aperture can be approximated by a Gaussian with the following standard deviation [24]:

$$
\sigma_{\mathrm{A}}=\frac{0.42 \lambda W_{\mathrm{D}}}{2 r_{\mathrm{ph}}} .
$$

This convolution has a well-known result (from any treaty on Gaussian error propagation combining Gaussians with top-hat uncertainty distributions):

$$
\sigma^{2}=\frac{1}{3}\left(\delta^{2}+3 \sigma_{A}^{2}(1-\theta(\mathrm{F}))\right) .
$$

$\theta(\mathrm{F})$ is the Heaviside step function, which ensures that the diffraction effect only appears for $\mathrm{F}<1$.

Additionally, there will be an effect on the standard deviation stemming from the inhomogeneity of the intensity profile along the perpendicular direction. The intensity value, at big enough distances from the axis of symmetry, decreases significantly. However, if the nozzlepinhole distance is much bigger than the pinhole radius, the intensity profile at the pinhole is approximately constant. This is often used in literature, where a center line intensity is calculated and then multiplied by the surface of the detector [14]. For the case of helium microscopes, where the nozzle-pinhole distance is typically of the order of several centimetres and the pinhole radius is of the order of a few micrometres, this approximation can always be used and eq. (4) suffices.

We define the focal spot size of the beam as the full width at half maximum associated to the standard deviation of the beam's intensity profile:

$$
\begin{aligned}
\Phi=2 \sqrt{2 \ln 2 / 3} \sqrt{\delta^{2}+} & 3 \sigma_{A}^{2}(1-\theta(\mathrm{F})) \\
& \equiv K \sqrt{\delta^{2}+3 \sigma_{A}^{2}(1-\theta(\mathrm{F}))} .
\end{aligned}
$$

For micrometer resolutions, we will see that due to the small wavelength of a helium beam, the Fresnel number is typically bigger than one (see Appendix A for a detailed description of the focal spot formula choice). This allows a purely geometrical optics treatment of the focal spot size. However, we choose to develop a formula that covers both extreme cases because sometimes the optimization procedure involves a wide span of Fresnel numbers.

From eq. (5) one can find three relations between $r_{\mathrm{S}}, a$ and $r_{\mathrm{ph}}$ (see Appendix B for the derivation). We consider two of these expressions, $r_{\mathrm{ph}} \rightarrow r_{\mathrm{ph}}\left(a, r_{\mathrm{S}}\right)$ and $a \rightarrow a\left(r_{\mathrm{ph}}, r_{\mathrm{S}}\right)$ :

$$
\begin{array}{r}
K^{2}(1+\gamma)^{2} r_{\mathrm{ph}}^{4}+2 K^{2} r_{\mathrm{S}} \gamma(1+\gamma) r_{\mathrm{ph}}^{3}+\left(K^{2} \gamma^{2} r_{\mathrm{S}}^{2}-\Phi^{2}\right) r_{\mathrm{ph}}^{2} \\
+K^{2}(1-\theta(\mathrm{F})) \epsilon=0 . \quad(6)
\end{array}
$$

Where $\gamma=\frac{W_{\mathrm{D}}}{a}$ and $\epsilon=3 W_{\mathrm{D}}^{2}(0.42 \lambda / 2)^{2}$. And:

$$
\begin{array}{r}
a^{2}\left(\frac{\Phi^{2}}{K^{2}}-r_{\mathrm{ph}}^{2}-3 \sigma_{A}^{2}(1-\theta(\mathrm{F}))\right)-a \cdot 2 r_{\mathrm{ph}} W_{\mathrm{D}}\left(r_{\mathrm{ph}}+r_{\mathrm{S}}\right) \\
-W_{\mathrm{D}}^{2}\left(r_{\mathrm{ph}}+r_{\mathrm{S}}\right)^{2}=0 .
\end{array}
$$

The equation (6) for the pinhole radius is a quartic equation in the Fraunhofer regime because the same focal spot size can be obtained when the diffraction term dominates over the geometrical term and vice-versa. In practice, the equation gives two real, positive solutions for which the one with maximum pinhole radius and therefore maximum intensity is chosen.

For the case of $a$ (eq. (7)), the solution is uni-evaluated because there exist only one positive root:

$$
\begin{aligned}
& a= \\
& \frac{r_{\mathrm{ph}} W_{\mathrm{D}}}{A}\left(r_{\mathrm{ph}}+r_{\mathrm{S}} \pm \sqrt{\left(r_{\mathrm{ph}}+r_{\mathrm{S}}\right)^{2}+A\left(1+2 \frac{r_{\mathrm{S}}}{r_{\mathrm{ph}}}+\frac{r_{\mathrm{S}}^{2}}{r_{\mathrm{ph}}^{2}}\right)}\right) .
\end{aligned}
$$

Where $A=\frac{\Phi^{2}}{K^{2}}-r_{\mathrm{ph}}^{2}-3 \sigma_{A}^{2}(1-\theta(\mathrm{F}))$, if $A$ is negative, $a$ is also negative or non-real (except the case when the square root is 0 ) and if $A$ is positive, we must take the positive root to ensure a positive value of $a$.

Using eq. (6) or (7), the system can be reduced to a two-variable optimization system.

\section{B. The intensity models}

As discussed in the introduction, the most general model of the intensity field of a supersonic helium beam is the ellipsoidal quitting surface model, with an elliptical velocity distribution:

$$
\begin{aligned}
f_{\text {ell }}(\vec{v})= & n\left(\frac{m}{2 \pi k_{\mathrm{B}} T_{\|}}\right)^{\frac{1}{2}}\left(\frac{m}{2 \pi k_{\mathrm{B}} T_{\perp}}\right) . \\
& \exp \left(-\frac{m}{2 k_{\mathrm{B}} T_{\|}}\left(v_{\|}-\bar{v}\right)^{2}-\frac{m}{2 k_{\mathrm{B}} T_{\perp}} v_{\perp}^{2}\right) .
\end{aligned}
$$

Where $m$ is the mass of a helium atom, $k_{\mathrm{B}}$ is Boltzmann's constant and $T_{\|}$and $T_{\perp}$ are the parallel and perpendicular temperatures. $\bar{v}$ is the most probable velocity of the beam along the radial direction and $v_{\|}$and $v_{\perp}$ are the parallel and perpendicular components of the velocity, corresponding to the radial and angular components in spherical coordinates.

Integrating eq. (9) over the quitting surface, across the skimmer and over the pinhole surface, one obtains the following intensity equation:

$$
\begin{array}{r}
I_{\mathrm{D}}=\frac{\tau I_{0}}{2 \pi a^{2} R_{\mathrm{F}}^{2} \mathrm{~L}} \int_{0}^{r_{\mathrm{ph}}} \int_{0}^{r_{\mathrm{S}}} \int_{0}^{\pi} g(\delta) r \cdot \rho \cos ^{3} \beta \cdot \epsilon^{3} \\
e^{-S^{2}\left(1-\epsilon^{2} \cos ^{2} \theta\right)} D(b) d \rho d r d \alpha .
\end{array}
$$


Where $R_{\mathrm{F}}$ is the radius of the quitting surface and $S=$ $\bar{v} / \sqrt{2 k_{\mathrm{B}} T_{\|} / m}$ is the parallel speed ratio. $I_{0}$ is the total intensity stemming from a nozzle of diameter $d_{\mathrm{n}}$ [13]:

$$
I_{0}=\kappa \frac{P_{0}}{k_{\mathrm{B}} T_{0}} \sqrt{\frac{2 k_{\mathrm{B}} T_{0}}{m}}\left(\frac{\pi}{4} d_{\mathrm{n}}^{2}\right) \sqrt{\frac{\gamma}{\gamma+1}}\left(\frac{2}{\gamma+1}\right)^{1 /(\gamma-1)} .
$$

Where $P_{0}$ and $T_{0}$ are the thermodynamic pressure and temperature of the helium gas before it undergoes the expansion. $\gamma=\mathrm{C}_{\mathrm{P}} / \mathrm{C}_{\mathrm{V}}=5 / 3$ for helium. $\kappa=2$ is a peak factor as defined in [13]. All other parameters are defined in detail in Appendix C. Unfortunately, the ellipsoidal quitting surface model has no simple analytical solutions and is often slow to compute over a wide space of solutions.

Using the quitting surface model with a single radial velocity distribution it can be shown that for $S \geq$ $5, r_{\mathrm{S}} \ll R_{\mathrm{F}}-x_{\mathrm{S}}$, the intensity arriving at a small pinhole reads [14]:

$$
I_{\mathrm{S}}=\frac{I_{0} \pi r_{\mathrm{ph}}^{2}}{\left(R_{\mathrm{F}}+a\right)^{2}}\left(1-\exp \left[-S^{2}\left(\frac{r_{\mathrm{S}}\left(R_{\mathrm{F}}+a\right)}{R_{\mathrm{F}}\left(R_{\mathrm{F}}-x_{\mathrm{S}}+a\right)}\right)^{2}\right]\right) .
$$

Where $R_{\mathrm{F}}-x_{\mathrm{S}}$ is the axial distance between the quitting surface and the tip of the skimmer. This equation was obtained independently by Sikora and Andersen [14] and thus we refer to it as Sikora's approximation as mentioned in the introduction.

We can use this equation together with eq. (6) for $\mathrm{F} \gg 1$ to obtain a simple analytical equation for the position of the intensity maximum, given either $a$ or $r_{\mathrm{S}}$ is taken to be constant.

$$
\nabla I_{\mathrm{S}}\left(r_{\mathrm{S}}, a\right)=(0,0), \quad \partial_{a}^{2} I<0, \quad \partial_{r_{\mathrm{S}}}^{2} I<0 .
$$

Which corresponds to a subset of the solutions of the following equation:

$$
\begin{array}{r}
\nabla \frac{r_{\mathrm{ph}}^{2}}{\left(R_{\mathrm{F}}+a\right)^{2}}\left(1-\exp \left[-S^{2}\left(\frac{r_{\mathrm{S}}\left(R_{\mathrm{F}}+a\right)}{R_{\mathrm{F}}\left(R_{\mathrm{F}}-x_{\mathrm{S}}+a\right)}\right)^{2}\right]\right) \\
=0 .
\end{array}
$$

Where the radius of the pinhole, for $F \gg 1$ can be obtained from eq. (6), which reduces to a quadratic equation with a single physical solution:

$$
r_{\mathrm{ph}}=\frac{\frac{\Phi a}{K}-W_{\mathrm{D}} r_{\mathrm{S}}}{a+W_{\mathrm{D}}} .
$$

From eq. (14), it can be shown that the maximum of intensity is at (see Appendix D):

$$
r_{\mathrm{S}}^{\max }=\frac{\Phi a}{2 W_{\mathrm{D}} K},
$$

as long as the following condition holds:

$$
\left(S \frac{R_{\mathrm{F}}+a}{R_{\mathrm{F}}\left(R_{\mathrm{F}}-x_{\mathrm{S}}+a\right)}\right)^{2}\left(\frac{\Phi a}{W_{\mathrm{D}} K} r_{\mathrm{S}}-r_{\mathrm{S}}^{2}\right) \ll 1 .
$$

The radius of the pinhole at the intensity maximum is then:

$$
r_{\mathrm{ph}}^{\max }=\frac{\Phi a}{2 K\left(a+W_{\mathrm{D}}\right)} \approx \frac{\Phi}{2 K}, \quad a \gg W_{\mathrm{D}} .
$$

Comparing with eq. (15) one sees the importance of condition (17), because if $W_{\mathrm{D}} r_{\mathrm{S}} \ll \frac{\Phi a}{K}$ and $a \gg W_{\mathrm{D}}$ is imposed, from eq. (15) one obtains:

$$
r_{\mathrm{ph}} \approx \frac{\Phi}{K} .
$$

Which corresponds to the case where the beam does not widen after the pinhole.

Unfortunately, no analytical solution has been found for $a^{\max }$, neither in this limit nor in the general case. However, the value can be easily obtained numerically (see Section III). The triplet $\left(a^{\max }, r_{\mathrm{ph}}^{\max }, r_{\mathrm{S}}^{\max }\right)$ is the optimal solution for the design of a helium microscope of a given resolution $\Phi$, given working distance, $W_{\mathrm{D}}$, and given conditions at the supersonic expansion $P_{0}, T_{0}$.

\section{RESULTS AND DISCUSSION}

\section{A. An example: $\Phi=5 \mu \mathrm{m}, W_{\mathrm{D}}=3 \mathrm{~mm}$.}

To illustrate the optimization method we consider the pinhole helium microscope presented by Barr et al [22]. This microscope has been successfully built and has produced some of the best helium microscopy images at a resolution of $5 \mu \mathrm{m}$. We evaluate how the microscope could be optimized using the same resolution and working distance as in the original setup.

By means of eq. (6), the problem is restricted to two variables: the skimmer-pinhole distance $a$ and the skimmer aperture $r_{\mathrm{S}}$. The intensity is then calculated by means of the ellipsoidal quitting surface model, eq. (10). A clear maximum of intensity can be observed which smoothly decreases along the line described by eq. (16) (see Fig. 2), giving the subset of maximums of intensity for each fixed $a$ value. The Fresnel number is plotted at Fig. 3. We see that in this case, the maximum is situated at $\mathrm{F}=19.42$, reasonably within the geometrical approximation.

It is also interesting to compare the intensity given by the ellipsoidal quitting surface model to the intensity given by Sikora's approximation. Both models peak at slightly different intensities, therefore it is useful to plot the fraction of the normalized intensities.

$$
\zeta=\frac{I_{\mathrm{S}} \cdot \max \left(I_{\mathrm{D}}\right)}{I_{\mathrm{D}} \cdot \max \left(I_{\mathrm{S}}\right)} .
$$

From Fig. 4 we see that Sikora's approximation fits well the behaviour of the quitting surface model near the intensity peak. Sikora's approximation diverges from the ellipsoidal quitting surface model at larger skimmers as expected theoretically, as it is in this regime that the component of the perpendicular temperature starts being important. 


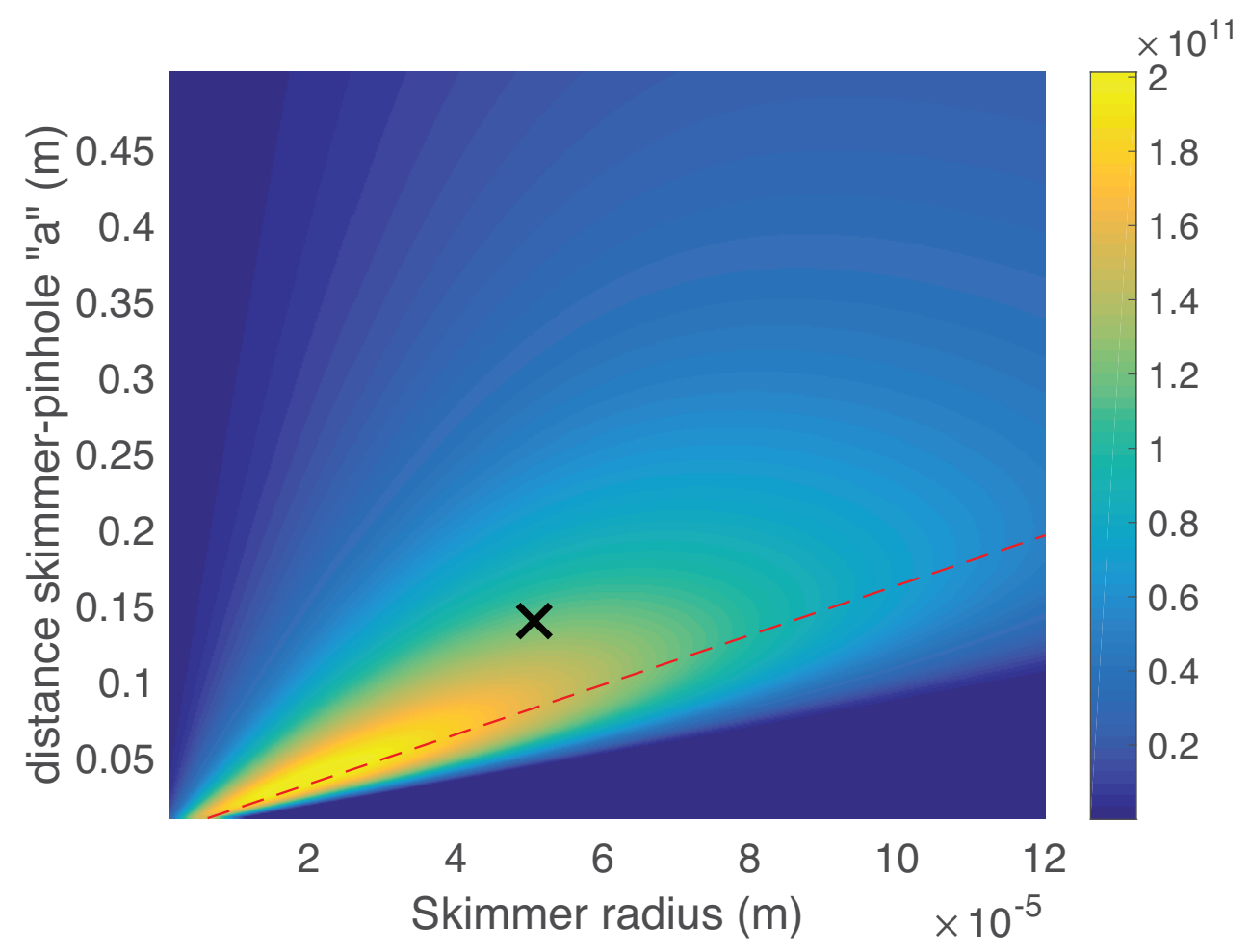

FIG. 2. Intensity (part/s) crossing a focal spot $(\Phi=5 \mu \mathrm{m})$ of a pinhole helium microscope for a span of values of $r_{\mathrm{S}}$ and $a$. The dashed line shows the subset of maximal solutions given by eq. (16). The intensity was calculated using the ellipsoidal quitting surface model with the following parameters: $T_{\|}=0.00802 \mathrm{~K}, T_{\perp}=0.00209 \mathrm{~K}, R_{\mathrm{F}}=0.0112 \mathrm{~m}, x_{\mathrm{S}}=0.0113 \mathrm{~m}, T_{0}=309 \mathrm{~K}$, $P_{0}=161$ Bar. The working distance of the microscope is set at $0.003 \mathrm{~m}$, and the nozzle diameter $d_{\mathrm{n}}$ at $10 \mu \mathrm{m}$. The maximum lies at $\left(a^{\max }, r_{\mathrm{ph}}^{\max }, r_{\mathrm{S}}^{\max }\right)=\left(0.035 \mathrm{~m}, 1.7886 \cdot 10^{-6} \mathrm{~m}, 2.0250 \cdot 10^{-5} \mathrm{~m}\right)$. The black cross indicates the configuration corresponding to the microscope designed by Barr et al [22]. The optimized design would increase the intensity by $75 \%$. However, a distance of only $35 \mathrm{~mm}$ between the two pinholes would not be so easy to realize experimentally with a good pumping speed.

\section{B. Parametric dependences}

The optimisation presented in Fig. 2 was performed for a room temperature supersonic expansion beam, as in the original experiment. We compare now with a cold beam, which can be obtained by cooling the nozzle. Cooling the beam increases the flow and changes the velocity distribution. For the cold beam calculation we choose the temperature $150 \mathrm{~K}$ because this is a temperature which can easily be kept stable using for example liquid nitrogen cooling. The position of the maximum changes significantly. The optimized $a^{\max }$ value decreases from $0.035 \mathrm{~m}$ to $0.026 \mathrm{~m}$, and the optimized skimmer radius decreases from $20.2 \mu \mathrm{m}$ to $14.7 \mu \mathrm{m}$. The pinhole radius at the maximum remains nearly constant (see Fig. 5).

The pinhole microscope system shows a clear maximum for a particularly defined supersonic expansion. However, the position of the intensity maximum varies with the expansion parameters. Such dependences can be easily evaluated using Sikora's approximation.

The dependence of the intensity maxima with the speed ratio follows the expected trend. Higher speed ratios favour smaller microscopes and skimmer openings (see Fig. 6) due to the weaker divergence of the beam. This also yields higher maximum intensity values. One sees that the pinhole size depends very weakly of the speed ratio as predicted by eq. (18). The radius of the skimmer depends on the speed ratio through the value of $a$ (see eq. (16)).

The intensity decreases and the skimmer-pinhole distance increases strongly with the working distance, $W_{\mathrm{D}}$. In other words, the working distance should always be chosen as small as possible (see Fig. 7). The optimum pinhole radius decreases with the working distance, because it is the main contributor to the widening of the beam. The skimmer radius shows a more complicated dependence, reaching a minimum at low working distances and then increasing smoothly. This must be understood in combination with the skimmer-pinhole distance $a$, which at small working distances is very small and favours a small skimmer radius to lower the divergence of the beam (see Fig. 7).

Regarding the dependences with the focal spot size $\Phi$, the intensity shows the expected quadratic dependence, similar to the area of a disk. This quadratic relation makes it hard to design high resolution microscopes with 


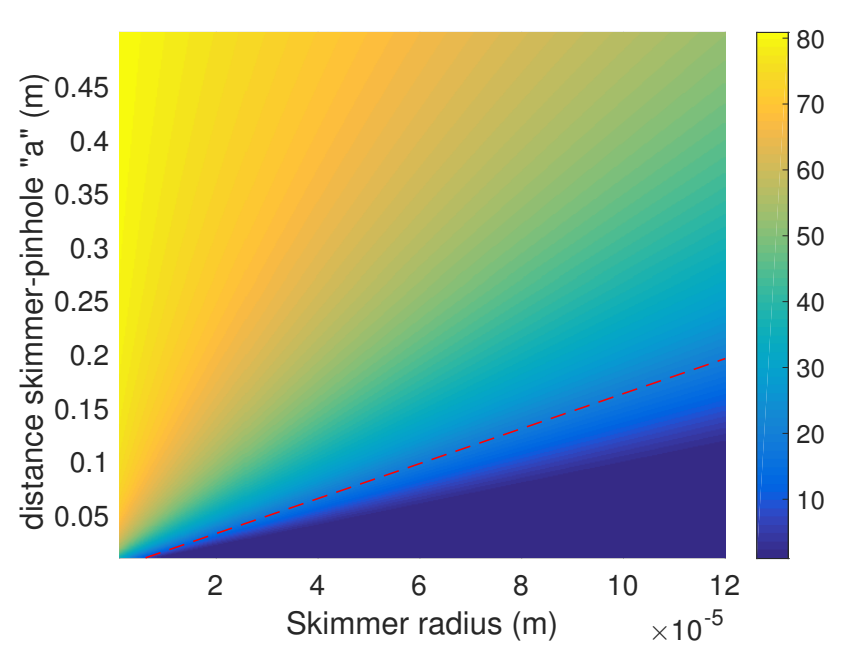

FIG. 3. Fresnel number for a span of values of $r_{\mathrm{S}}$ and $a$. The Fresnel number was calculated assuming an average wavelength of $\bar{\lambda}=8.2535 \cdot 10^{-11} \mathrm{~m}$. Note that the line of maximal intensity given by eq. (16) spans a region where $F \gtrsim 10$. The working distance of the microscope is set to $3 \mathrm{~mm}$, and the resolution to $5 \mu \mathrm{m}$.

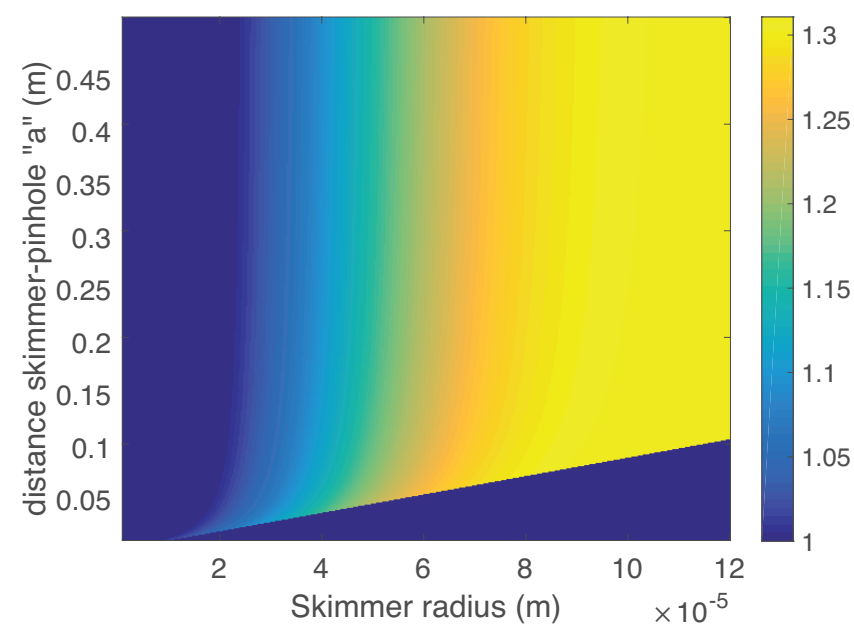

FIG. 4. Sikora's approximation intensity divided by the ellipsoidal model, $\zeta$ for a span of values of $r_{\mathrm{S}}$ and $a$ (eq. (20)). Note how both models diverge at high apertures due to the omission of the perpendicular temperature effect in Sikora's approximation. At the intensity maximum both models behave similarly with $\zeta=0.9722$.

enough intensity (see Fig. 8). Both aperture radii increase with the focal spot, the pinhole does it quasi linearly following eq. (16) while the skimmer shows a more complicated dependence. The skimmer-pinhole distance $a$ peaks at small focal spot sizes and then decreases monotonically as the influence of the airy diffraction disappears (see Fig. 8).

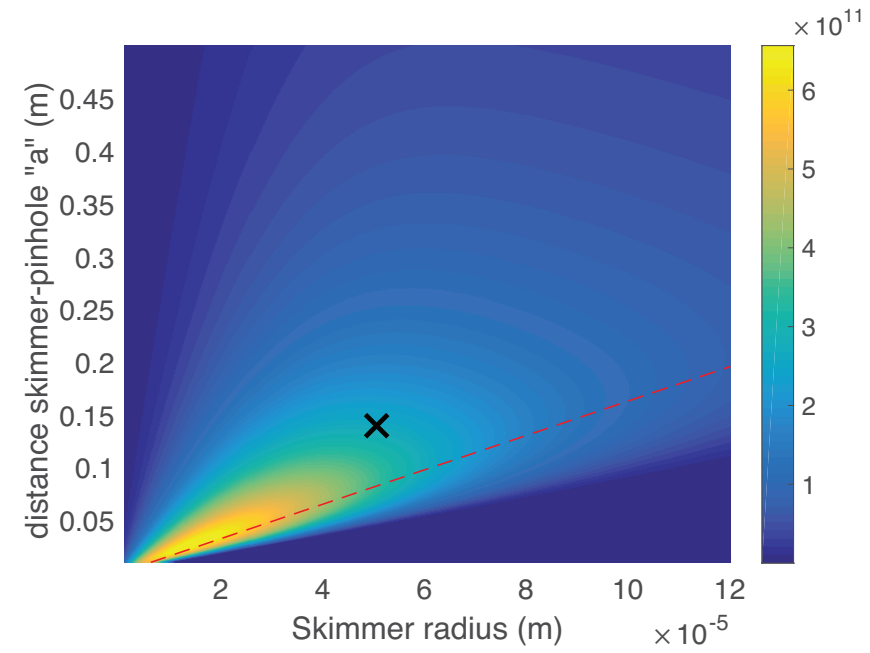

FIG. 5. Intensity (part/s) crossing the focal spot of a pinhole helium microscope for a span of values of $r_{\mathrm{S}}$ and $a$. The dashed line shows the subset of maximal solutions given by eq. (16). The intensity was calculated using the ellipsoidal quitting surface model with the following parameters: $T_{\|}=0.0054 \mathrm{~K}, T_{\perp}=0.0039 \mathrm{~K}, R_{\mathrm{F}}=0.0112 \mathrm{~m}$, $x_{\mathrm{s}}=0.0113 \mathrm{~m}, T_{0}=131.3 \mathrm{~K}, P_{0}=161$ Bar. The working distance of the microscope is set to $3 \mathrm{~mm}$, and the resolution to $5 \mu \mathrm{m}$. The maximum lies at $\left(a^{\max }, r_{\mathrm{ph}}^{\max }, r_{\mathrm{S}}^{\max }\right)=$ $\left(0.0260,1.7714 \cdot 10^{-6}, 1.4750 \cdot 10^{-5}\right) \mathrm{m}$. The black cross indicates the configuration corresponding to the microscope designed by Barr et al [22].

\section{Realistic resolution limits}

Using Sikora's approximation, one can easily obtain the resolution limit of a realisable pinhole helium microscope. A minimal count rate of 100 counts per second is chosen and compared with the expected signal for the optimal microscope at each resolution. Lambertian reflection [25] at $\pi / 4$ radians relative to the sample normal is assumed with the detector opening area perpendicular to the reflected direction. The intensity seen at a given solid angle is given by Lambert cosine law:

$$
I_{\Omega}=I_{\mathrm{inc}} \cos (\pi / 4) \Omega_{\mathrm{D}}=I_{\mathrm{inc}} \cos ^{2}(\pi / 4) \frac{\pi R^{2}}{d^{2}} .
$$

Where $I_{\text {inc }}$ is the incident intensity per solid angle, which corresponds to $I_{\mathrm{s}} / \pi$. $\Omega_{\mathrm{D}}$ is the solid angle seen by a detector with a circular opening of radius $R$ at a distance $d$, oriented as described above. By considering the efficiency of the detector, the formula giving the expected count-rate reads:

$$
N=\frac{I_{\mathrm{s}}^{o p t i m} R^{2}}{d^{2}} \eta \cos \left(\frac{\pi}{4}\right)^{2} \approx 1.4 \cdot 10^{-7} I_{\mathrm{s}}^{\text {optim }} .
$$

Where $R$ is the detector radius, chosen to be $0.5 \mathrm{~mm}$. $d$ is the distance between the sample surface and the detector, set at $3 \mathrm{~mm}$. This corresponds to the solid angle of 0.02 $\pi$ sr mentioned in the abstract. $I_{\mathrm{s}}^{\text {optim }}$ is the optimized 


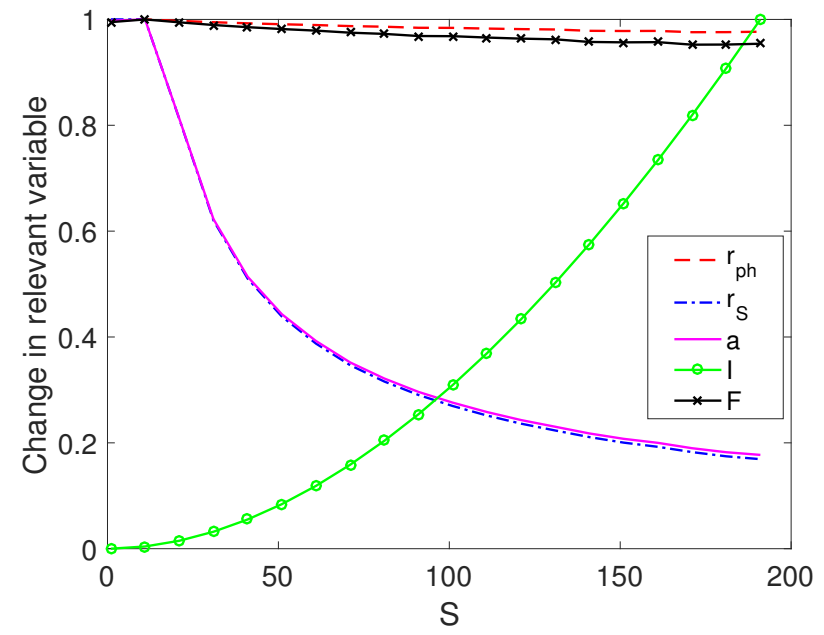

FIG. 6. Normalised optimized parameters of a pinhole helium microscope for a span of values of the speed ratio $S$. The intensity was calculated using Sikora's approximation with the following parameters: $T_{0}=131.3 \mathrm{~K}, P_{0}=161 \mathrm{Bar}$. The working distance of the microscope is set at $0.003 \mathrm{~m}$.

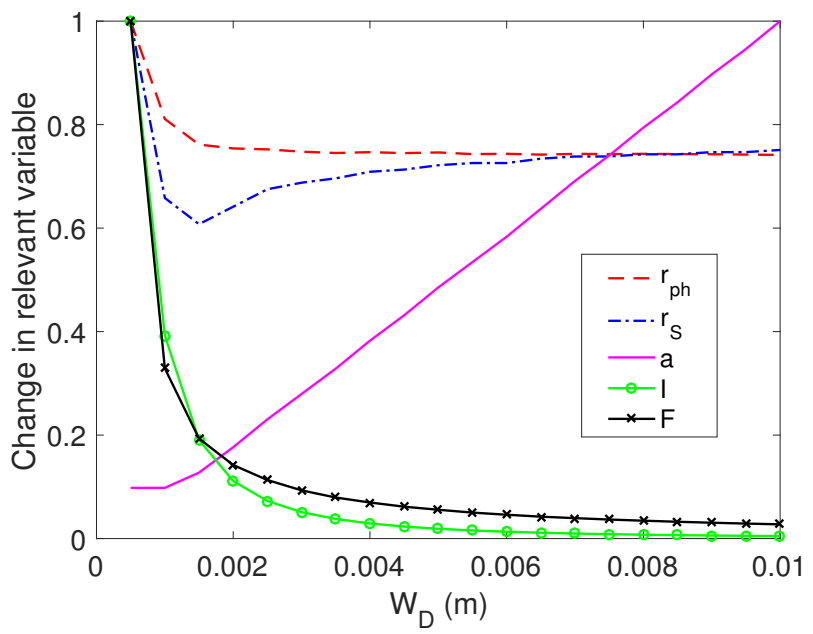

FIG. 7. Normalised optimized parameters of a pinhole helium microscope for a span of values of the working distance $W_{\mathrm{D}}$. The intensity was calculated using Sikora's approximation with the following parameters: $T_{0}=131.3 \mathrm{~K}, P_{0}=161$ Bar. The working distance of the microscope is set at 0.003 $\mathrm{m}$.

intensity using Sikora's approximation. $\eta$ is the efficiency of the detector, chosen to be between $10^{-3}[26-29]$ and $10^{-5}$ (an upper limit estimate for a typical, commercial mass spectrometer). Note that all parameters are set as in the paper by Barr et al. [22]. As we can see in Fig. 9, sub-micrometer resolutions are achievable with a reasonable count rate.

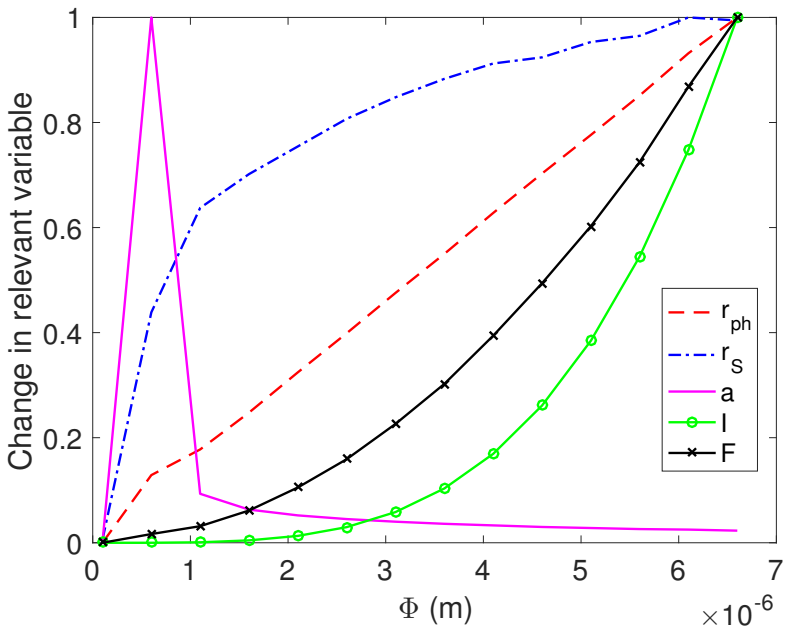

FIG. 8. Normalised optimized parameters of a pinhole helium microscope for a span of values of the focal spot $\Phi$. The intensity was calculated using Sikora's approximation with the following parameters: $T_{0}=131.3 \mathrm{~K}, P_{0}=161$ Bar. The working distance of the microscope is set at $0.003 \mathrm{~m}$.

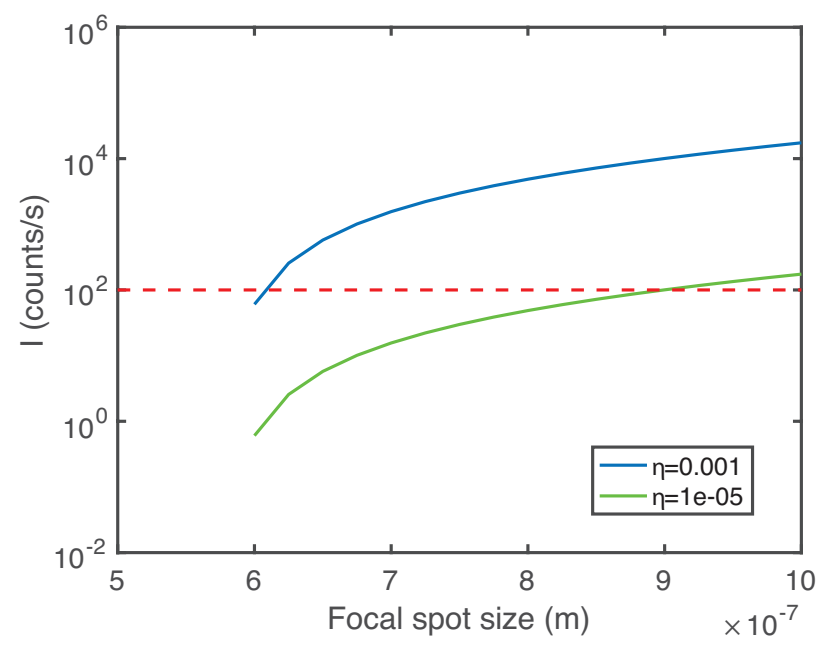

FIG. 9. Optimized count rate for different focal spot sizes. The intensity was calculated using Sikora's approximation with the following parameters: $T_{0}=131.3 \mathrm{~K}, P_{0}=161 \mathrm{Bar}$. The working distance of the microscope is set at $0.003 \mathrm{~m}$. The efficiency of the detector, placed at $\pi / 4$ radians is $\eta=1 \cdot 10^{-5}$ (lower line) or $\eta=1 \cdot 10^{-3}$ (upper line).

\section{The small working distance limit}

A small $W_{\mathrm{D}}$ helium microscope would be suitable for the investigation of all samples currently investigated with scanning probe techniques as discussed in the introduction. In the limit $W_{\mathrm{D}} r_{\mathrm{S}} / a \ll r_{\mathrm{ph}}$, for $\eta=0.001$, the maximum of intensity is always higher than 100 counts per second for a collection solid angle of $0.02 \pi$ sr. Therefore, the minimal achievable resolution is determined by 
the theoretical trade-off between Airy diffraction and beam widening in eq. (5). The smallest achievable $\Phi$ is given by the point where decreasing further the pinhole radius does not improve the resolution any more, because the diffraction term dominates. For a working distance of $10 \mu \mathrm{m}$, this point corresponds to (see Appendix E):

$$
\Phi=K \sqrt{0.42 \lambda W_{\mathrm{D}} \sqrt{3}} \approx 33 \mathrm{~nm} .
$$

With $\lambda=8.25 \cdot 10^{-11} \mathrm{~m}$, corresponding to $T_{0}=$ $131.3 \mathrm{~K}$. This equation is derived under the assumption $W_{\mathrm{D}} r_{\mathrm{S}} / a \ll r_{\mathrm{ph}}$, which for the case of $W_{\mathrm{D}}=10 \mu \mathrm{m}$ only holds weakly, the real resolution is a bit higher, $40 \mathrm{~nm}$. This has been calculated setting a lower bound for $a$ of 5 $\mathrm{cm}$, chosen as a technical constraint for the placement of a pump in the skimmer-pinhole chamber. A helium pinhole microscope with a working distance of $10 \mu \mathrm{m}$ has already been demonstrated by Witham and Sanchez in their $350 \mathrm{~nm}$ resolution configuration [12].

\section{CONCLUSION}

We present a theoretical model of a neutral helium pinhole microscope using the quitting surface approach to model the source intensity. We show that for a given microscope resolution, working distance, and constant source characteristics, there is a unique optimized solution. This optimized solution gives the sizes of the two apertures of the system and their separation. Our pinhole microscope model can easily be adapted to other source models considering attenuation of the beam or describing the flow of other atoms or molecules. The adaptation is done simply by changing the intensity equation whilst using the same optical expressions. We show that with the quitting surface intensity source model, high intensity, helium pinhole microscopes with a resolution down to around $40 \mathrm{~nm}$ are realisable. Further we suggest that an intensity improvement of up to $75 \%$ for existing helium pinhole microscopes is theoretically possible (although a practical realisation can be challenging).

\section{APPENDIX A: THE FOCAL SPOT SIZE AT HIGH FRESNEL NUMBERS}

If the Fresnel number is sufficiently high, a ray-optics treatment is sufficient to describe the focal spot size. In such a treatment, no convolution with a diffraction function is needed and the beam has the form of a top-hat function. Therefore, in such cases the resolution of the microscope is best understood by redefining the constant $K$ in a way that the FWHM of the beam is simply the width of the top-hat function. This corresponds to $K \equiv 2$ so that eq. (5) reduces to:

$$
\Phi=2 \delta
$$

\section{APPENDIX B: DERIVATION OF EQ. (6) AND (7)}

Deriving eq. (6) and (7) is a simple algebra problem consisting of extracting $a \rightarrow a\left(r_{\mathrm{ph}}, r_{\mathrm{S}}\right)$ and $r_{\mathrm{ph}} \rightarrow$ $r_{\mathrm{ph}}\left(a, r_{\mathrm{S}}\right)$ from eq. (5). We shall begin by obtaining $r_{\mathrm{ph}} \rightarrow r_{\mathrm{ph}}\left(a, r_{\mathrm{S}}\right)$. Squaring eq. (5) one obtains:

$$
\begin{aligned}
\frac{\Phi^{2}}{K^{2}} & =\delta^{2}+3 \sigma_{\mathrm{A}}^{2}(1-\theta(F)) \\
& =r_{\mathrm{ph}}^{2}\left(1+\frac{W_{\mathrm{D}}}{a}\right)^{2}+2 r_{\mathrm{ph}}\left(1+\frac{W_{\mathrm{D}}}{a}\right) \frac{W_{\mathrm{D}} r_{\mathrm{S}}}{a} \\
& +\left(\frac{W_{\mathrm{D}} r_{\mathrm{S}}}{a}\right)^{2}+3\left(\frac{0.42 \lambda}{2}\right)^{2} \frac{W_{\mathrm{D}}^{2}}{r_{\mathrm{ph}}^{2}}(1-\theta(F)) .
\end{aligned}
$$

Defining $\gamma=\frac{W_{\mathrm{D}}}{a}$ and $\epsilon=3 W_{\mathrm{D}}^{2}(0.42 \lambda / 2)^{2}$, multiplying by $r_{\mathrm{ph}}^{2}$ and grouping by powers of $r_{\mathrm{ph}}$, one obtains eq. (6). To obtain eq. (7) we can use eq. (B1), and multiply by $a^{2}$ :

$$
\begin{aligned}
& \quad \frac{\Phi^{2}}{K^{2}} a^{2} \\
& \quad=a^{2} r_{\mathrm{ph}}^{2}\left(1+\frac{W_{\mathrm{D}}}{a}\right)^{2}+2 a^{2} r_{\mathrm{ph}}\left(1+\frac{W_{\mathrm{D}}}{a}\right) \frac{W_{\mathrm{D}} r_{\mathrm{S}}}{a} \\
& +a^{2}\left(\frac{W_{\mathrm{D}} r_{\mathrm{S}}}{a}\right)^{2}+3 a^{2}\left(\frac{0.42 \lambda}{2}\right)^{2} \frac{W_{\mathrm{D}}^{2}}{r_{\mathrm{ph}}^{2}}(1-\theta(F)) .
\end{aligned}
$$

Expanding the quadratic sums and grouping by powers of $a$, eq. (7) is recovered.

\section{APPENDIX C: THE ELLIPSOIDAL QUITTING SURFACE MODEL}

The ellipsoidal quitting surface model used in this paper is an extension of Sikora's ellipsoidal quitting surface model with the difference that the skimmer is allowed to be in a different position than the quitting surface. Such a model was published by U. Bossel in 1974 [19]. Unfortunately the original paper has a couple of typos so we describe here the corrected equations in detail. The main intensity equation arriving at the pinhole is eq. (10):

$$
\begin{array}{r}
I_{\mathrm{D}}=\frac{\tau I_{0}}{2 \pi a^{2} R_{\mathrm{F}}^{2} \mathrm{~L}} \int_{0}^{r_{\mathrm{ph}}} \int_{0}^{r_{\mathrm{S}}} \int_{0}^{\pi} g(\delta) r \cdot \rho \cos ^{3} \beta \cdot \epsilon^{3} \\
e^{-S^{2}\left(1-\epsilon^{2} \cos ^{2} \theta\right)} D(b) d \rho d r d \alpha .
\end{array}
$$

Where $R_{\mathrm{F}}$ is the radius of the quitting surface and $S=\bar{v} / \sqrt{2 k_{\mathrm{B}} T_{\|} / m}$ is the parallel speed ratio. $\epsilon \equiv$ $\left(\left(\tau \sin ^{2} \theta+\cos ^{2} \theta\right)^{-1 / 2}, \tau \equiv \frac{T_{\|}}{T_{\perp}}\right.$ are auxiliary functions. The function $\mathrm{D}(\mathrm{b})$ is defined as follows:

$D(b) \equiv \frac{2}{\sqrt{\pi}} b e^{-b^{2}}+\left(2 b^{2}+1\right)[1+\operatorname{erf}(b)], \quad b \equiv S \epsilon \cos \theta$. 
Where $I_{0}$ is defined in eq. (11). $g(\delta)$ is obtained from the angular term in eq. (12) of Beijerinck and Verster paper [13] (in Bossel's work a $\cos ^{2}$ function is chosen instead [19]):

$$
g(\delta)=\cos ^{3}\left(\frac{\pi \theta}{2 \theta_{0}}\right), \quad \theta_{0}=\frac{\pi}{2} \sqrt{\frac{\gamma+1}{\gamma-1}-1}=\frac{\pi}{2} .
$$

Where $\gamma=C_{\mathrm{P}} / C_{\mathrm{V}}=5 / 3$ for helium has been used. L corresponds to the integration of $g(\delta)$ along the half sphere (all the intensity emitted by the source is set to be contained in $g(\delta))$.

$$
L \equiv \int_{0}^{\frac{\pi}{2}} g(\delta) \sin \delta d \delta=\frac{1}{4}
$$

$\rho$ is the radial coordinate at the pinhole plane and $r$ is the radial coordinate at the skimmer plane. The angles $\beta, \alpha$ and $\theta$ are shown in Fig. 10. The analytical expressions that relate them to the radial coordinates of the system follow:

$$
\begin{gathered}
\cos \beta=\frac{a}{\sqrt{a^{2}+(r \sin \alpha)^{2}+(\rho-r \cos \alpha)^{2}}}, \quad a=x_{\mathrm{D}}-x_{\mathrm{S}} . \\
\cos \theta=\frac{x_{\mathrm{R}}\left(x_{\mathrm{D}}-x_{\mathrm{R}}\right)-y_{\mathrm{R}}^{2}+z_{\mathrm{R}}\left(\rho-z_{\mathrm{R}}\right)}{R_{\mathrm{F}} \sqrt{\left(x_{\mathrm{D}}-x_{\mathrm{R}}\right)^{2}+y_{\mathrm{R}}^{2}+\left(\rho-z_{\mathrm{R}}\right)^{2}}} .
\end{gathered}
$$

Where:

$$
\begin{gathered}
x_{\mathrm{R}}=x_{\mathrm{D}}-\xi\left(x_{\mathrm{D}}-x_{\mathrm{S}}\right), \quad y_{\mathrm{R}}=r \sin \alpha\left(\frac{x_{\mathrm{D}}-x_{\mathrm{R}}}{x_{\mathrm{D}}-x_{\mathrm{S}}}\right), \\
z_{\mathrm{R}}=r \cos \alpha\left(\frac{x_{\mathrm{D}}-x_{\mathrm{R}}}{x_{\mathrm{D}}-x_{\mathrm{S}}}\right)-\rho\left(\frac{x_{\mathrm{D}}-x_{\mathrm{R}}}{x_{\mathrm{D}}-x_{\mathrm{S}}}\right)+\rho, \quad(\mathrm{C} 7)
\end{gathered}
$$

are the Cartesian coordinates of a point $\mathbf{P}$ on the quitting surface (see Fig. 10).

$$
\begin{gathered}
\xi=\frac{B-\sqrt{B^{2}-A C}}{A} . \\
A \equiv\left(x_{\mathrm{D}}-x_{\mathrm{S}}\right)^{2}+(r \sin \alpha)^{2}+(\rho-r \cos \alpha)^{2}, \\
B=x_{\mathrm{D}}\left(x_{\mathrm{D}}-x_{\mathrm{S}}\right)+\rho(\rho-r \cos \alpha), \\
C=x_{\mathrm{D}}^{2}+\rho^{2}-R_{\mathrm{F}}^{2} .
\end{gathered}
$$

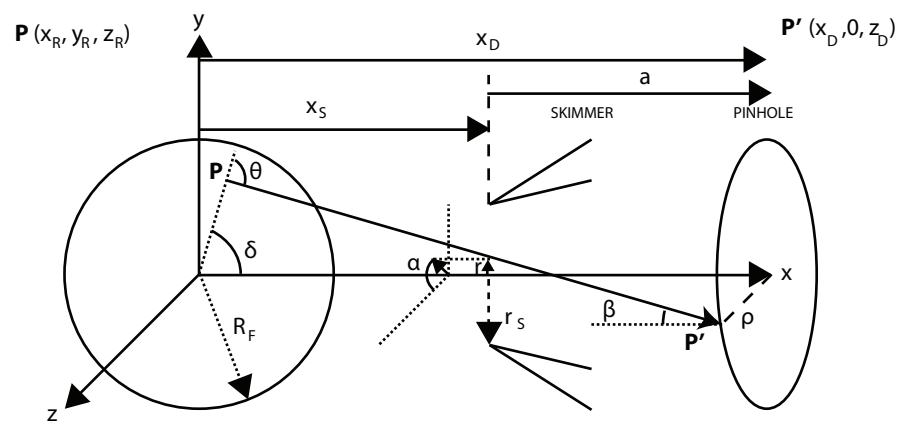

FIG. 10. Illustration of all variables used in the ellipsoidal quitting surface model. $\mathbf{P}$ is a point on the quitting surface from which a particle leaves in a straight trajectory until P', a point placed on the pinhole plane. The point on the quitting surface is given by the set of Cartesian coordinates $\left(x_{\mathrm{R}}, y_{\mathrm{R}}, z_{\mathrm{R}}\right)$, which can be related to the polar coordinates $r, \alpha, \rho$ for further integration. $x_{\mathrm{S}}$ is the distance from the nozzle to the skimmer and $x_{\mathrm{D}}$ is the distance from the nozzle to the pinhole. Therefore $a=x_{\mathrm{D}}-x_{\mathrm{S}}$. The angles $\beta$ and $\theta$ can also be expressed in terms of $r, \alpha$ and $\rho$.

\section{APPENDIX D: DERIVATION OF EQ. (16)}

To obtain eq. (16) we must differentiate $I_{\mathrm{S}}$ with respect to the skimmer radius and set it equal to 0 (eq. (12)). To do so we will first need to introduce eq. (15) in eq. (12) in order to obtain $I_{\mathrm{S}} \rightarrow I_{\mathrm{S}}\left(a, r_{\mathrm{S}}\right)$. Once this is done, defining $D^{2} \equiv\left(\frac{S\left(R_{\mathrm{F}}+a\right)}{R_{\mathrm{F}}\left(R_{\mathrm{F}}-x_{\mathrm{S}}+a\right)}\right)^{2}$ and taking the derivative, we obtain:

$$
\begin{aligned}
& 2 D^{2} r_{\mathrm{S}} e^{-D^{2} r_{\mathrm{S}}^{2}}\left(\frac{\Phi a}{K}-W_{\mathrm{D}} r_{\mathrm{S}}\right)^{2}- \\
& 2 W_{\mathrm{D}}\left(1-e^{-D^{2} r_{\mathrm{S}}^{2}}\right)\left(\frac{\Phi a}{K}-W_{\mathrm{D}} r_{\mathrm{S}}\right)=0,
\end{aligned}
$$

which reduces to:

$$
e^{-D^{2} r_{\mathrm{S}}^{2}}\left(2 D^{2} r_{\mathrm{S}}\left(\frac{\Phi a}{K}-W_{\mathrm{D}} r_{\mathrm{S}}\right)+2 W_{\mathrm{D}}\right)=2 W_{\mathrm{D}} .
$$

Taking the natural logarithm we get:

$$
D^{2} r_{\mathrm{S}}^{2}=\ln \left(1+D^{2}\left[\frac{\Phi a}{W_{\mathrm{D}} K} r_{\mathrm{S}}-r_{\mathrm{S}}^{2}\right]\right) .
$$

Now, if $D^{2}\left[\frac{\Phi a}{W_{\mathrm{D}} K} r_{\mathrm{S}}-r_{\mathrm{S}}^{2}\right] \ll 1$, we can expand the logarithm at first order using Taylor series. Thanks to eq. (15) we know that this condition corresponds to the case of small pinholes (or of small $D^{2}$ ).

$$
r_{\mathrm{S}}^{2} \approx \frac{\Phi a}{W_{\mathrm{D}} K} r_{\mathrm{S}}-r_{\mathrm{S}}^{2} \rightarrow r_{\mathrm{S}} \approx \frac{\Phi a}{2 W_{\mathrm{D}} K} .
$$

To proof that this corresponds to a maximum of the intensity function, we must show that the second derivative of eq. (12) respect the skimmer radius is negative. The 
second derivative evaluated at eq. (D4) reads (out of a positive constant $C^{2}$ ):

$$
\begin{gathered}
C^{2} \frac{d^{2}}{d r_{\mathrm{S}}^{2}} I_{\mathrm{S}}= \\
-\left(\frac{\Phi a D}{2 K}\right)^{2} e^{-D^{2} r_{\mathrm{S}}^{2}}\left[\frac{D^{2} \Phi^{2} a^{2}}{W_{\mathrm{D}}^{2} K^{2}}+6\right]+2 W_{\mathrm{D}}^{2}\left(1-e^{-D^{2} r_{\mathrm{S}}^{2}}\right)
\end{gathered}
$$

Where we have not evaluated $r_{\mathrm{S}}$ in the exponentials in purpose, because we want to use $D^{2}\left[\frac{\Phi a}{W_{\mathrm{D}} K} r_{\mathrm{S}}-r_{\mathrm{S}}^{2}\right] \approx$ $D^{2} r_{\mathrm{s}}^{2} \ll 1$. By combining this condition with eq. (D5), one obtains:

$$
C^{2} \frac{d^{2}}{d r_{\mathrm{S}}^{2}} I_{\mathrm{S}} \approx-\left(\frac{\Phi a D}{2 K}\right)^{2}\left[\frac{D^{2} \Phi^{2} a^{2}}{W_{\mathrm{D}}^{2} K^{2}}+6\right]<0
$$

\section{APPENDIX E: DERIVATION OF EQ. (23)}

One can derive the low limit working distance resolution limit directly from eq. (5):

$$
\Phi=K \sqrt{\delta^{2}+3 \sigma_{A}^{2}(1-\theta(\mathrm{F}))}
$$

At the low resolution limit (very small pinholes), $\mathrm{F}$ is smaller than 1 and diffraction terms are present. The working distance is assumed to be very small, i.e.:

$$
\frac{W_{\mathrm{D}} r_{\mathrm{S}}}{a} \ll r_{\mathrm{ph}}, \quad \frac{W_{\mathrm{D}}}{a} \ll 1 .
$$

Eq. (E1) is then reduced to:

$$
\Phi=K \sqrt{r_{\mathrm{ph}}^{2}+3 \sigma_{A}^{2}}=\sqrt{r_{\mathrm{ph}}^{2}+3\left(\frac{0.42 \lambda W_{\mathrm{D}}}{2 r_{\mathrm{ph}}}\right)^{2}} .
$$

One can find that $\frac{\partial}{\partial r_{\mathrm{ph}}} \Phi^{2}=0, \frac{\partial^{2}}{\partial^{2} r_{\mathrm{ph}}} \Phi^{2}>0$ if:

$$
r_{\mathrm{ph}}=\sqrt{\sqrt{3} \frac{0.42 \lambda W_{\mathrm{D}}}{2}} .
$$

Which gives, the minimum resolution limit:

$$
\Phi=K \sqrt{0.42 \lambda W_{\mathrm{D}} \sqrt{3}}
$$

[1] G. Bracco and B. Holst, "Surface Science Techniques," (Springer, 2013).

[2] D. Farias, G.H Rieder Rep. Prog. Phys. 61, 1575-1664 (1998).

[3] M. Koch, S. Rehbein, G. Schmahl, T. Reisinger, G. Bracco, W. E. Ernst, and B. Holst, J. Microsc. 229, 1 (2008).

[4] S. D. Eder, T. Reisinger, M. M. Greve, G. Bracco, and B. Holst, New. J. Phys. 14, 73014 (2012).

[5] O. Carnal, M. Sigel, T. Sleator, H. Takuma, and J. Mlynek, Phys. Rev. Lett. 67, 3231 (1991).

[6] B. Holst and W. Allison, Nature 390, 244 (1997).

[7] R. B. Doak, R. E. Grisenti, S. Rehbein, G. Schmahl, J. P. Toennies, and C. Wöll, Phys. Rev. Lett. 83, 4229 (1999).

[8] P. Witham and E. Sanchez, Rev. Sci. Instrum. 82, 103705 (2011).

[9] P. Witham and E. Sanchez, Cryst. Res. Technol. 29, 690 (2014).

[10] A. Fahy, M. Barr, J. Martens, and P. Dastoor, Rev. Sci. Instrum 86, 023704 (2015).

[11] M. Barr, A. Fahy, J. Martens, A. P. Jardine, D. J. Ward, J. Ellis, W. Allison, and P. C. Dastoor, Nat. Commun. 7, 10189 (2016).

[12] P. Witham and E. Sanchez, J. Microsc. 248, 223 (2012).

[13] H. C. W. Beijerinck and N. F. Verster, Physica C 111, 327 (1981).
[14] G. S. Sikora, Analysis of asymptotic behavior of free jets: Prediction of Molecular Beam Instensity and Velocity Distributions, Ph.D. thesis, Princeton (1973).

[15] J. Toennies and K. Winkelmann, J. Chem. Phys. 66, 3965 (1977).

[16] T. Reisinger, G. Bracco, S. Rehbein, G. Schmahl, W. E. Ernst, and B. Holst, J. Phys. Chem A 111, 12620 (2007).

[17] L. Pedemonte and G. Bracco, J. Chem. Phys. 119, 1433 (2003).

[18] S. D. Eder, G. Bracco, T. Kaltenbacher, and B. Holst, J. Phys. Chem A 118, 4 (2014).

[19] U. Bossel, Skimming of Molecular Beams from Diverging Non-equilibrium Gas Jets, Deutsche Luft- und Raumfahrt. Forschungsbericht (Deutsche Forschungs-und Versuchsanstalt für Luft-und Raumfahrt, 1974).

[20] H. C. W. Beijerinck, R. J. F. V. Gerwen, E. R. T. Kerstel, J. F. M. Martens, E. J. W. V. Vliembergen, M. Smits, and G. H. Kaashoek, Chem. Phys. 96, 153 (1985).

[21] H. Hedgeland, A. P. Jardine, W. Allison, and J. Ellis, Rev. Sci. Instrum. 76, 123111 (2005).

[22] M. Barr, A. Fahy, A. Jardine, J. Ellis, D. Ward, D. A. Maclaren, W. Allison, and P. C. Dastoor, Nucl. Instrum. Methods B 340, 76 (2014).

[23] E. Hecht, Optics, edited by A. Black, Vol. 4 (Addison Wesley, 2002).

[24] T. Stewart McKechnie, General Theory of Light Propagation and Imaging Through the Atmosphere, 1st ed. 
(Springer, 2016) p. 624.

[25] J. H. Lambert, Photometria, sive De mensura et gradibus luminus, colorum et umbrae (1760).

[26] M. Matsuki, Company MB Scientific, Private communication, detector available on a commercial basis upon request.
[27] M. DeKieviet, D. Dubbers, M. Klein, U. Pieles, and C. Schmidt, Rev. Sci. Instrum. 71, 123301 (2000).

[28] A. R. Alderwick, A. P. Jardine, H. Hedgeland, D. A. MacLaren, W. Allison, and J. Ellis, Rev. Sci. Instrum. 79 (2008), 10.1063/1.3030858.

[29] W. Allison, Private Communication 